\title{
Philonsorbonne
}

13 | 2019

Année 2018-2019

\section{Droit naturel, contractualisme et dissension chez Thomas Hobbes et Emer de Vattel}

\section{Lyess BOUDERBALA}

\section{(2) OpenEdition}

\section{Journals}

\section{Édition électronique}

URL : https://journals.openedition.org/philonsorbonne/1069

DOI : 10.4000/philonsorbonne.1069

ISSN : 2270-7336

\section{Éditeur}

Publications de la Sorbonne

\section{Édition imprimée}

Date de publication : 30 janvier 2019

Pagination : 11-38

ISSN : 1255-183X

\section{Référence électronique}

Lyess BOUDERBALA, « Droit naturel, contractualisme et dissension chez Thomas Hobbes et Emer de Vattel », Philonsorbonne [En ligne], 13 | 2019, mis en ligne le 03 février 2019, consulté le 11 juin 2021. URL : http://journals.openedition.org/philonsorbonne/1069; DOI : https://doi.org/10.4000/ philonsorbonne.1069 


\title{
Droit naturel, contractualisme et dissension chez Thomas Hobbes et Emer de Vattel
}

\author{
Lyess BOUDERBALA
}

\section{Introduction}

Du désaccord théorique à la révolution, en passant par la désobéissance et la guerre civile, les phénomènes de dissension politique prennent des formes multiples. La proposition de remèdes contre les actes par lesquels un ou plusieurs individus contredisent l'ordre politique occupe une place considérable dans la philosophie politique. Qu'il s'agisse, en amont, de contrôler la diffusion des opinions sur lesquelles de tels actes se fondent ou, en aval, de punir la contestation lorsqu'elle est formalisée, les stratégies proposées sont nombreuses. En témoigne le nombre de concepts que la philosophie n'a cessé de produire, tout particulièrement de l'Antiquité à la Modernité, pour en traduire un aspect particulier : les Grecs avec la stásis, le tumulto des Romains, Thomas d'Aquin et le péché de la sédition ou encore la discorde chez Machiavel, etc ${ }^{1}$.

L'objectif plus restreint de cette contribution est de mesurer l'écart qui sépare Thomas Hobbes et Emer de Vattel quant au traitement des phénomènes de dissension politique. Alors qu'on trouve chez le premier l'affirmation selon laquelle "aucun homme en possession de la puissance souveraine ne peut être mis à mort en toute justice, ou puni de quelque façon

1. Sur l'absence et le manque d'une «stasiologie», voir G. AGAMBEN, La Guerre civile : pour une théorie politique de la stasis, traduit par Joël Gayraud, Paris, Éditions Points, « Points », 2015, vol. 1, 76 p. 
que ce soit, par ses sujets $»^{2}-$ conséquence nécessaire de l'institution de la République par laquelle les hommes répondent à l'obligation qu'ils ont de se conserver -, le second répond que «s'il [le souverain] se rend le fléau de l'état, il se dégrade lui-même ; ce n'est plus qu'un ennemi public, contre lequel la nation peut et doit même se défendre : et s'il a porté la tyrannie à son comble, pourquoi même la vie d'un ennemi si cruel et si perfide seraitelle épargnée ? » ${ }^{3}$. La distance entre les deux ne paraît pas pouvoir être plus grande.

Hobbes et Vattel s'accordent pourtant à la fois sur le rôle du droit naturel et sur l'utilité du pacte social. La puissance souveraine est instituée par un acte juridique à travers lequel le sujet s'engage et dont il retire, en contrepartie, une tranquillité inestimable. Et le droit naturel, déduit à partir de la seule raison, fournit des règles irréfutables au-delà desquelles l'action du souverain ne peut aller.

Outre leur détermination divergente des droits de la souveraineté, la confrontation entre ces deux auteurs permet, nous semble-t-il, de révéler que leurs positions opposées sur le statut du souverain, vulnérable ou invulnérable - c'est-à-dire sur la liberté d'action des sujets en cas de mécontentement - s'expliquent par leur compréhension de la dissension. Réduite au cas de la désobéissance et de la résistance, nous montrerons que leur conception du conflit interne s'écarte autant sur l'essence de celui-ci (ruine de l'État / division de l'État), que sur son origine (passion de vanité / souci de justice) et sa finalité (récupération illégale d'un droit abandonné / récupération de la souveraineté). Alors que chez Hobbes, la contestation ne peut être admise qu'à un niveau individuel comme réapparition ponctuelle du droit naturel lorsque le souverain intente à la vie d'un sujet - et dès lors qu'elle est collective, l'opposition au souverain est l'œuvre d'une multitude sans unité qui entend créer fictivement du pouvoir -, pour Vattel, à l'inverse, la résistance des sujets peut être la condition par laquelle ils récupèrent la souveraineté transmise au prince ou en font apparaître une nouvelle.

Cette comparaison, outre qu'elle permette de rapporter la singularité des compréhensions hobbesienne et vattelienne de la dissension politique au contexte historique de leurs auteurs, donne l'occasion de révéler que si Vattel s'écarte autant de Hobbes, c'est en raison du statut attribué aux rapports entre les souverains. Avec Hobbes, l'État est replié sur lui-même, car hors de ses frontières subsiste un état de nature similaire à la condition des individus en l'absence d'une puissance commune, position qui prend tout son sens lorsqu'on la rapporte à la préoccupation de l'auteur pour la

2. T. HobBes, Léviathan ou Matière, forme et puissance de l'État chrétien et civil, traduit par Gérard Mairet, Paris, Gallimard, « Folio/Essais », 2000, II, XVIII, p. 296.

3. E. VAtTel, Le Droit des gens, ou Principes de la loi naturelle appliqués à la conduite et aux affaires des nations et des souverains, Neuchâtel, Suisse, Société typographique, t. 1, I, IV, § 51, p. 66. 
stabilité interne ${ }^{4}$. Or Vattel, qui soutient l'existence d'un droit qui s'applique entre les nations, droit capable de les contraindre même lorsqu'elles se font la guerre, est conduit à en défendre l'application y compris lorsqu'un souverain punit des rebelles. De la sorte, le diplomate suisse peut, contrairement à Hobbes, voir dans la dissension interne une reconfiguration du politique et non plus sa négation.

En outre, la restitution de l'examen de la dissension par Hobbes et Vattel fait résonner le traitement du désaccord politique avec les débats qui occupent l'Âge classique. La nouvelle méthode de déduction du droit naturel et le développement du contractualisme ${ }^{5}$ renouvellent la formulation des phénomènes de contestation. En effet, puisque toute opposition à la volonté du souverain consiste dans la tentative d'échapper à un commandement, cela suppose que le contestataire puisse invoquer une norme supérieure à celle imposée par son souverain. La contestation, qu'elle prenne la forme d'une désobéissance ou d'une résistance, suppose une divergence entre des corpus de normes distincts ${ }^{6}$. Or, c'est précisément ce que les doctrines modernes du droit naturel vont proposer en déduisant à partir de la nature humaine un ensemble d'obligations immuables et universelles capables par conséquent de s'imposer face au droit civil produit par la volonté contingente du souverain ${ }^{7}$. Par ailleurs, la construction de l'État à partir de l'engagement volontaire du particulier paraît paralyser la possibilité, pour ce dernier, de s'extraire du lien d'obligation dont il est la seule source. L'engagement du sujet, par lequel il charge son représentant de produire le droit civil auquel il promet d'obéir, implique (selon les conditions du contrat) que tout phénomène de dissension publique revienne pour le sujet à tenter de se soustraire à une soumission dont il a convenu. Le droit naturel n'étant plus déduit d'un ordre du monde rapporté à la volonté et la raison divines, et l'État ne s'expliquant plus par un naturalisme ou une théologie de droit $\operatorname{divin}^{8}$, la signification de la dissension ne peut qu'en être transformée.

4. On ne peut rien dire des relations entre souverains. En effet, les États demeurent guidés par leur droit du glaive car ils évoluent, du fait de l'analogie domestique, dans un état de nature à plus grande échelle. Cf. T. HobBes, Léviathan, op. cit., I, XIII, p. 227.

5. Pour décrire l'engouement du XVII ${ }^{\mathrm{e}}$ siècle pour la théorie du contrat, Simone GoyardFabre parle d' « inflation contractualiste », cf. S. GOYARD-FABRE, L'interminable querelle du contrat social, Ottawa, Éd. de l'Université d'Ottawa, «Philosophica », 1983, p. 177-178.

6. S'opposer à l'injonction d'un souverain nécessite de mobiliser une autre obligation issue d'un corps supérieur de droits. Sur ce point voir le début de l'article de É. DESMONS, « Droit de résistance et histoire des idées », in Pouvoirs, $\mathrm{n}^{\circ} 4, \mathrm{~N}^{\circ} 155,4$ décembre 2015, p. 29-40.

7. Utiliser le droit naturel comme étalon du droit civil n'est évidemment pas une invention de la modernité. La nouveauté réside dans la déduction de ce droit qui se fait désormais à partir d'une définition de l'homme et non plus d'un Dieu transcendant. En conséquence, tout recours à la doctrine paulinienne du pouvoir s'en trouve fragilisée. Sur l'histoire de l'opposition entre droit naturel et droit positif, voir l'ouvrage de L. STRAUSS, Droit naturel et histoire, traduit par Monique Nathan et Éric de Dampierre, Paris, Flammarion, «Champs », 1986, chap. 3.

8. J. TERREL, Les Théories du pacte social : droit naturel, souveraineté et contrat de Bodin à Rousseau, Paris, France, Éd. du Seuil, 2001. 


\section{Première partie - La naissance du corps politique}

\section{L'arrière-plan}

\section{a. Droit naturel et contradiction entre les puissances}

Alors que Charles $\mathrm{I}^{\mathrm{er}}$ poursuit, après le règne de son père, l'ambition de gouverner en monarque absolu - c'est-à-dire sans parlement - quitte à susciter un mécontentement croissant dans cette Angleterre vieille de plus de deux siècles de tradition parlementaire, Hobbes prend position dès 1640 pour l'indivisibilité de la souveraineté 9 . Or, parce qu'il se place du côté des royalistes, futurs perdants de la guerre civile qui découlera de l'opposition entre partisans du roi et parlementaires, ce conflit constitue pour lui une expérience bien personnelle.

L'essence de la guerre civile selon Hobbes est de révéler sans détour le bellicisme de la nature humaine, raison pour laquelle il s'efforce de concevoir une autorité politique à même de s'en prémunir qui soit donc fondée sur cette même nature. La philosophie politique réclame une anthropologie qui doit en constituer le premier moment ${ }^{10}$. En témoigne la construction de la république qui, dans les Éléments, se situe dans la première partie consacrée aux hommes en tant que personnes naturelles ${ }^{11}$. Par ce geste Hobbes insiste sur la nécessité pour la science politique de tirer ses conclusions à partir d'une étude de l'homme tel qu'il est, d'où sa «mise à nu » grâce au dispositif méthodologique de l'état de nature.

La condition de l'homme s'y résume aux traits principaux suivants : les individus s'affrontent pour augmenter leur puissance de sorte à accroître leur capacité à obtenir ce qu'il leur apparaît comme un bien. Or, du fait de la possibilité, pour la puissance d'un individu, de se faire puissance d'un autre, chacun s'efforce de faire de chaque autre un moyen supplémentaire pour parvenir à ses fins.

9. T. HobBEs, Éléments de la loi naturelle et politique, traduit par Dominique Weber, Paris, Librairie générale française, 2003, II, 1, § 16, p. 236-237. 1640 est une année de troubles pour l'Angleterre. Charles $1^{\text {er }}$ est décidé à unifier la politique du royaume et à harmoniser les pratiques religieuses de l'Angleterre et de l'Écosse. Il s'attire de ce fait les foudres des Écossais qui refusent de se plier à l'anglicanisme, et du Parlement anglais, opposé à l'idée d'une loi unique. On comprend que Hobbes consacre un chapitre entier au cas de la rébellion, dont cinq paragraphes portent exclusivement sur les opinions qui conduisent à la ruine de l'État, à savoir celles qu'en Angleterre les puritains et parlementaires diffusent.

10. Hobbes indique son objectif sans détour. Cf. T. HoBBES, Éléments de la loi naturelle et politique, op. cit., I, I, $\$ 1$, p. 80 : «L'explication vraie et claire des éléments des lois naturelles et politiques, qui est ma visée présente, dépend de la connaissance de ce qu'est la nature humaine, de ce qu'est un corps politique $[\ldots] »$.

11. Concernant la place de l'état de nature, Ninon Grangé souligne l'hésitation de Hobbes, manifestée par l'écart que le Léviathan introduit par rapport au De Cive. Cf. N. GRANGE, « La cité en guerre. Violence naturelle ou violence politique ? Un aspect de la guerre civile chez Hobbes », in Cahiers d'études italiennes, n 1, 15 novembre 2004, p. 17-38, p. 18. 
Cette compétition est rendue nécessaire du fait de la mobilisation par chacun du droit naturel premier par lequel tout homme est autorisé à faire tout ce que sa puissance lui permet pour se conserver ${ }^{12}$. La rivalité entre les hommes découle alors des tentatives illimitées qu'ils sont en droit de mettre en œuvre pour se prémunir de tout désavantage et pour éviter «par-dessus tout ce terrible ennemi de la nature, la mort de laquelle nous attendons à la fois la perte de toute puissance et aussi la plus grande des douleurs corporelles accompagnant cette perte ${ }^{13}$.

De plus, l'utilisation de sa puissance propre est une obligation. Si le droit de nature est un pouvoir de faire, l'exercice de ce pouvoir est rendu obligatoire par la première loi de nature qui, bien qu'elle commande à la recherche de la paix, ordonne le recours à la "guerre ${ }^{14}$ lorsque celle-ci n'est pas assurée. Ainsi, même si la raison, stimulée par la crainte de la mort, découvre les règles générales par lesquelles la paix peut advenir, l'homme découvre également qu'il lui est interdit de nuire à sa propre vie, donc de ne pas faire usage de son premier droit de nature, qui lui permet de faire tout ce qui est en son pouvoir pour se conserver tant que sa sécurité n'est pas garantie. Par conséquent, l'autorité de la loi de nature, qui donne à chacun un droit à s'approprier toutes les choses, y compris « le corps des autres » 15 , fait de cette rivalité interhumaine, renforcée par l'égalité naturelle entre les hommes, une nécessité. Les hommes ne peuvent que s'assujettir ou s'éliminer.

\section{b. L'état de nature n'est pas un état de guerre}

Pour Vattel, la question n'est pas tellement celle du fondement juridique et rationnel du corps politique mais plutôt celle du caractère contraignant des devoirs que les souverains ont les uns envers les autres, autrement dit, non pas les conventions entre les particuliers mais celles entre les États. Ainsi, au lieu d'une construction théorique en vase close de l'État comme on la trouve chez Hobbes, Vattel se consacre plus directement à l'exposé du droit des gens ${ }^{16}$. Son expérience professionnelle est sur ce point éclairante. Grâce à l'invitation du comte de Brühl, premier ministre de l'électeur de Saxe Auguste III, il devient son conseiller d'ambassade à Dresde, puis son

12. Ce droit fait de chacun l'unique juge pour lui-même des moyens nécessaires pour préserver son intégrité corporelle de la destruction.

13. T. HobBES, Éléments, op. cit., I, XIV, § 6, p. 178.

14. T. HoBBES, Léviathan, op. cit., I, XVI, p. 231.

15. Ibid.

16. Chez Vattel, le corpus du droit des gens intègre des règles déterminant les relations entre nations et entre les sujets, car il est l'application du droit naturel entre les États. De même que les individus sont obligés de suivre les principes du droit naturel lorsqu'ils interagissent, de même les nations ont pour obligation de se soumettre au caractère obligatoire du droit naturel qui, pour elles, se nomme droit des gens. Cependant, le contenu diffère car le droit naturel doit être adapté aux nations. Ce droit se découpe en droit des gens naturel et droit des gens positif. Et lorsqu'il est positif, ce droit se constitue selon des modes distincts qui en changent la nature : droit des gens volontaire, conventionnel ou coutumier. 
ministre à Berne. Et la guerre de Sept ans - guerre qui du fait des alliances et des empires coloniaux dépasse les seules Prusse et Autriche -, pendant laquelle il conseille l'électeur de Saxe, lui fait saisir l'importance de cette science du droit des gens dont l'objet est de circonscrire, par le droit, les rapports entre les nations.

On comprend qu'il soit, dans la perspective de ce diplomate, inutile de s'attarder sur la fiction de l'état de nature. C'est précisément à l'occasion d'une remarque sur cet état que l'on trouve l'une des rares mentions de Hobbes chez Vattel ${ }^{17}$. Contre Hobbes, il affirme que la guerre ne peut être naturelle à l'homme puisque sa nature est, non pas ce qu'il est, mais ce vers quoi il doit tendre ${ }^{18}$.

Or, loin de présenter une description fouillée de cet état ${ }^{19}$, Vattel ne le mobilise ni pour justifier le «passage » à l'état civil, ni pour en penser les propriétés ${ }^{20}$. Au lieu d'une longue description de la condition des hommes dans cet état, Vattel préfère amorcer son ouvrage par la définition du corps politique ou, plus précisément des corps politiques, pluriel qui trahit une préoccupation pour les rapports entre sociétés politiques : «Les nations ou États, sont des corps politiques, des sociétés d'hommes unis ensemble pour procurer leur salut et leur avantage, à forces réunies. ${ }^{21}$.

Vattel ne s'appesantit pas sur la création de l'État, il insiste plutôt sur son dessein qui n'est autre que le salut de ses membres. L'association entre les hommes a pour fin de leur offrir une plus grande capacité à se prémunir contre les lésions qui peuvent leur être faites. À la place du droit de nature hobbesien, Vattel pose le droit de sûreté qui répond à l'obligation, dictée par la nature aux hommes comme aux nations, de se conserver et de se perfectionner. Mais avec ce droit il ne s'agit pas uniquement de conservation de soi mais aussi de défense contre les injustices. Or, parce qu'il est parfait, ce droit permet de contraindre par la force les autres à effectuer le devoir qui

17. Vattel mentionne très peu Hobbes dans ses œuvres, attitude d'autant plus surprenante lorsqu'on la compare à celle des juristes qui le précèdent ou lui sont contemporains (Pufendorf, Burlamaqui, Thomasius, Wolff, etc.), chez lesquels la référence au philosophe anglais est récurrente. À titre d'exemple, dans son ouvrage majeur, Le Droit des gens, Hobbes n'est mentionné (sauf erreur de notre part) qu'à trois reprises.

18. E. de VATTEL, Le Droit des gens, op. cit., t. 2, IV, I, § 1, p. 209 : « Hobbes a osé dire que la guerre est l'état naturel de l'homme. Mais si, comme la raison le veut, on entend par l'état naturel de l'homme, celui auquel il est destiné et appelé par sa nature, il faut dire plutôt que la paix est son état naturel ».

19. Ce qui est d'autant plus surprenant comparé à Pufendorf qui propose plusieurs états de nature, et lui consacre un chapitre entier, cf. S. von PUFENDORF, Le Droit de la nature et des gens, ou Système général des principes les plus importants de la morale, de la jurisprudence et de la politique, traduit par Jean Barbeyrac, Reproduction en fac-Similé., Caen, Centre de philosophie politique et juridique, «Bibliothèque de philosophie politique et juridique », 1989 , t. 1 , I, I, § 7-11; t. 1, II, II.

20. Il faut excepter le droit de punir dont la conservation conduirait à retourner à l'état de nature.

21. E. de Vattel, Le Droit des gens, op. cit., t. 1, Prélim., § 1, p. 25. Cette définition est reprise au singulier au premier paragraphe du premier chapitre. 
y répond. Le remplacement du droit sur toute chose hobbesien par le droit de sûreté renforce donc la possibilité de se protéger contre les lésions, et ce par trois voies d'action : la défense, l'obtention d'une réparation ou la punition $^{22}$.

Ainsi, réduire le dessein de la société politique à la seule sécurité n'est plus satisfaisant. Sa fin est bien plus large, elle doit garantir aux sujets les conditions nécessaires au bonheur en leur fournissant «la commodité et les agréments de la vie », en protégeant la propriété privée, et en leur assurant des réparations au cas où ils subiraient des injures ${ }^{23}$.

\section{Fondation du politique}

\section{a. Institution du souverain et obligation politique}

Pour Hobbes, l'état de nature ne fait pas que révéler le caractère généralisé de la violence en l'absence d'autorité politique. La contradiction entre les puissances, qui constitue l'essence de cet état, indique paradoxalement la voie à suivre pour qu'il ne puisse jamais advenir. Puisqu'un individu peut augmenter sa puissance en subordonnant celle d'un autre à la sienne, il faut alors assujettir toutes les puissances à une seule pour que disparaisse le risque d'une opposition. L'issue réside donc dans la formation de la puissance la plus grande qui soit, « composée des puissances de la plus grande partie des hommes unis par consentement en une personne naturelle ou civile qui possède l'usage de toutes leurs puissances, usage dépendant de sa volonté ${ }^{24}$.

Le mécanisme d'institution du souverain est tout tracé. Il n'est autre que le tiers au profit duquel chacun s'engage envers chaque autre à ne plus faire usage de son droit à toute chose. Cet abandon ${ }^{25}$, consistant dans le fait de «se défaire de la liberté que l'on a d'empêcher un autre de profiter du droit qui est le sien sur cette même chose ${ }^{26}$, réclame que le souverain soit exclu des conventions, et identique pour tous. Son extériorité est la condition

22. Haggenmacher ajoute que ces trois possibilités correspondent aux trois finalités de la guerre juste admises par Vattel, P. HAGGENMACHER, «Le modèle de Vattel et la discipline $\mathrm{du}$ droit international », in Vincent Chetall et Peter HAgGenMacher (dir.), Vattel's international law in a XXIst century perspective, Martinus Nijhoff Publishers, Leiden, Pays-Bas, Boston, États-Unis d'Amérique, 2011, p. 35.

23. E. de VATTEL, Le Droit des gens, op. cit., I, II, § 15, p. 44-45. La société politique doit permettre aux hommes de connaître une vie heureuse, de jouir paisiblement de ce qui leur appartient et de connaître une plus grande tranquillité. Voir aussi Ibid., I, 2, § 15. Le bonheur n'est pas, comme chez Hobbes, réduit au fait de maintenir ouverte la voie des désirs.

24. T. HobBEs, Léviathan, op. cit, I, X, p. 171.

25. Pour le rapport entre abandon et transfert chez Hobbes voir M. PECHARMAN, « Le contrat comme transfert volontaire de droit» in Gregory LEwKOWICZ et Mikhaïl XIFARAS (dir.), Repenser le contrat, Paris, France, Dalloz, 2009, 39-54.

26. T. HobBES, Léviathan, op. cit., I, XIV, p. 232. 
de son pouvoir de contrainte. En effet, parce qu'il ne participe pas aux conventions que les sujets passent entre eux, le tiers souverain conserve son droit de nature, et nul ne peut le contraindre. Libre de faire usage de toute sa puissance, - puissance qui inclut celle de tous les sujets -, il peut donc garantir la sécurité de tous grâce au droit de punir. Et parce qu'il est identique pour tous, il assure le passage de la multiplicité à l'unité des individus. Chacun et tous désignent le même tiers, de sorte à composer une personne civile dotée d'une volonté unique dans laquelle les volontés distinctes sont incluses.

Dans les Éléments l'extériorité du souverain par rapport au pacte est rapportée au modèle du dessaisissement. Se dessaisir de son droit revient pour le sujet à s'engager à ne pas lui résister lorsqu'il mobilisera ses facultés corporelles ou cognitives ${ }^{27}$. Dans le Léviathan, c'est le concept d'«autorisation ${ }^{28}$ associé à celui de «personne» ${ }^{29}$ qui fonde une obligation positive d'obéissance. L'autorisation dépasse la non-résistance, car elle engage le sujet à s'identifier à la volonté du tiers souverain, faisant de ses décisions celles du sujet. Puisque chacun s'engage à faire sien les actes futurs de ce tiers dont l'unité produit celle de la multitude représentée, id est du peuple, alors le souverain agit au nom de tous, et ses décisions valent pour tous et pour chacun ${ }^{30}$.

Unique détenteur du droit de juger des moyens nécessaires à l'avènement de la paix entre les individus, le souverain décide de "ce qu'il faut faire et de ce qu'il ne faut pas faire dans la vie quotidienne ${ }^{31}$, c'est-àdire des lois civiles. En plus de sa fonction de législateur, son rôle est directement pensé en rapport avec le risque de la dissension puisqu'il a pour mission d'empêcher la querelle interne en contrôlant les opinions publiques. En situant la dissension entre les sujets dans une différence entre des conceptions diverses du «meum et du tuum, du juste et de l'injuste, du bon et du mauvais $»^{32}$, Hobbes se donne les moyens de faire du souverain le

27. T. HobBES, Éléments, op. cit., I, XIX, § 10, p. 224 : «Et parce que nul ne peut réellement transférer sa propre force [strength] à un autre, ou cet autre la recevoir, il faut comprendre que transférer sa puissance et sa force [strength] n'est pas autre chose que de se défaire ou de se dessaisir de son droit propre de résister au bénéficiaire du transfert ». Dans le De Cive, le transfert de droit est également rapporté à la non-résistance ; cf. T. Hobbes, Du Citoyen, trad. Philippe Crignon, Paris, Flammarion, 2010, I, II, § 4, p. 111 : « [Quant au droit de tous sur toutes choses], le transfère à autrui celui qui [...] déclare à cet autre [...] vouloir que désormais il ne lui soit plus permis de résister à celui-là lorsqu'il fera certaines choses, quand il pouvait auparavant lui résister légitimement. Que le transfert de droit consiste uniquement dans la non résistance, cela se déduit du fait qu'avant le transfert de droit, celui à qui on le transfère avait déjà alors un droit sur toutes choses ».

28. Cf. T. HobBes, Léviathan, op. cit., II, XVII, p. 288.

29. Ibid., I, XVI, p. 270.

30. T. Hobbes, Éléments, op. cit., I, XII, § 8, p. 168 : «Lorsque plusieurs volontés sont impliquées ou incluses dans la volonté d'un seul ou de davantage qui consentent [...], alors cette implication de plusieurs volontés en un seul ou en davantage est appelée "UNION" ".

31. T. HobBes, Du Citoyen, op. cit., II, VI, § 9, p. 171, voir aussi Éléments, op. cit., II, I, § 10, p. 231-232, et Léviathan, op. cit., II, XIX, p. 479.

32. T. HobBes, Éléments, op. cit., p. 230. 
responsable unique des jugements privés sur le bien et le mal ${ }^{33}$. Chacun doit suivre les lois civiles qui sont les seules règles morales, et nul ne peut opposer une morale privée au souverain. Et puisque tout acte est une suite de volitions naissant d'une délibération à propos d'une opinion, le souverain, qui est censeur, contrôle les actions des sujets et donc les conditions de la concorde ${ }^{34}$.

Ce point se justifie chez Hobbes par une identification entre le vrai et le rationnel. La vérité d'une doctrine est réduite à sa conformité avec la paix, paix commandée par la raison. Puisque la recherche de la paix constitue l'essence de la loi de nature, loi identifiée à la recta ratio, tout contenu de pensée qui l'entrave est donc irrationnel, c'est-à-dire faux ${ }^{35}$.

\section{b. L'autonomie du pacte d'association}

À la démonstration géométrique de Hobbes sur l'institution du souverain, Vattel oppose une supposition. Il faut présumer que la constitution de l'État réclame la mise en commun du droit de sûreté des hommes de sorte à produire une autorité publique capable de pallier à leur faiblesse à l'état de nature ${ }^{36}$.

L'absence d'une longue description de la sortie de l'état de nature ${ }^{37}$ n'empêche pas l'auteur de proposer un modèle singulier en ce qu'il défend la primauté de l'association entre les particuliers sur la soumission qu'ils contractent avec leur souverain. Contre Hobbes, pour qui l'association entre les individus est en même temps une soumission au souverain ${ }^{38}$, Vattel

33. Il n'y a pas pour Hobbes d'éthique objective car le bien et le mal ne sont que des qualités relatives au sujet sentant, dérivées des passions que sont l'appétit et l'aversion. Dire d'une action qu'elle est bonne ne dit rien de l'action mais du rapport du sujet à celle-ci. Il s'agit d'une relation interne et subjective.

34. Sur ce point voir B. Binoche, Religion privée, opinion publique, Paris, Vrin, 2012, p. 26-29.

35. T. HobBes, Léviathan, op. cit., I, XVIII, p. 296-297. On comprend que Hobbes soumette le théologique à la même exigence de paix. De par leur éternité et leur universalité, les lois de nature sont divines. Ainsi, il y a correspondance: les lois divines confirment celles de la nature, et le souverain est également en charge de l'interprétation des Écritures.

36. E. de VAtTel, Le Droit des gens, op. cit., t. , Prélim., § 10, p. 30.

37. De même, la fondation de l'État et l'institution du souverain sont peu détaillées du fait du souci principal de Vattel qui est l'application du droit des gens et non la constitution du droit civil.

38. À ce propos Simone Goyard-Fabre affirme : «Il est assez vain de se demander s'il s'agit, selon la terminologie usitée depuis le Moyen Âge, d'un pactum associationis ou d'un pactum subjectionis. Dans la logique de Hobbes, il y a soumission des citoyens à la contrainte légale parce que tous les individus ont remis d'un commun consentement leurs droits de nature dans les mains du Léviathan : s'ils ne se soumettaient pas à la loi civile, ils se contrediraient en violant le mandat qu'ils ont confié à leur représentant : celui-ci n'accomplit en effet que les actions dont les citoyens sont les auteurs. Il n'y a donc pas chez Hobbes deux pactes, l'un d'association, l'autre, subséquent de soumission. La fondation de l'État entraîne ipso facto l'obéissance à la loi ». Cf. S. GOYARD-FABRE, L'Interminable querelle du contrat social, Ottawa, Éd. de l'Université d'Ottawa, «Philosophica », 1983, p. 171. 
distingue deux actes d'association aux finalités distinctes. D'abord, une association entre les individus, un pactum associationis qui crée l'État et son autorité, et ensuite, si ces derniers le souhaitent, un pactum subjectionis qui transfère la puissance de commander à un souverain auquel ils se soumettent.

La thèse du juriste est que l'État est constitué dès que des particuliers se regroupent et composent une volonté supérieure. L'association des individus dispose d'une stabilité propre qui ne dépend pas de l'élection d'un souverain. À l'inverse de Hobbes pour lequel l'unité de la personne du souverain fonde celle des représentés, Vattel défend l'antériorité et l'autonomie de la souveraineté sur la personne du souverain à qui elle peut, à titre de possibilité, être remise. L'obéissance fondamentale, celle qui produit de l'obligation politique, est celle par laquelle les individus s'associent entre eux et se soumettent au tout constitué ${ }^{39}$.

La supposition suivante résume l'association première: «chaque particulier s'est engagé envers tous à procurer le bien commun, et tous se sont engagés envers chacun à lui faciliter les moyens de pourvoir à ses besoins, à le protéger et à le défendre ${ }^{40}$. L'engagement n'a pas lieu de chacun à chacun, mais de chacun envers tous. En abandonnant le souci de leur conservation à la nation, les particuliers produisent une autorite ${ }^{41}$ qui « appartient originairement et essentiellement au corps même de la société, auquel chaque membre s'est soumis et a cédé les droits qu'il tenait de la nature $»^{42}$. De ce pacte résulte une obligation mutuelle : les individus doivent protéger la nation, qui elle est chargée de les conserver grâce à son autorité.

Cette autorité publique donne au concept de souveraineté son contenu. En effet, la souveraineté est rapportée à ce pouvoir « qui commande dans la société civile, qui ordonne et dirige ce que chacun doit y faire pour en atteindre le but ${ }^{43}$, pouvoir qui doit, et c'est là un point fondamental, être indépendant de toute influence externe. Écrivant dans le contexte d'une Europe consumée par les conquêtes ${ }^{44}$, le diplomate helvète ne réduit pas

39. En ce sens l'association suffit pour rendre compte de l'État. Sur ce point voir Emmanuelle JOUANNET, Emer de Vattel et l'émergence doctrinale du droit international classique, Paris, A. Pédone, 1998, p. 323 : «Ignorant la thèse du double contrat, il fait naître la société civile du seul pacte associatif car il pense que celle-ci forme une personne morale du seul fait de la règle majoritaire qui permet de dégager une volonté unique du peuple assemblée. En adoptant la thèse de la souveraineté originaire du peuple, il défend donc l'idée de la création simultanée du corps social et de l'État ».

40. E. de VAtTel, Le Droit des gens, op. cit., t. 1, I, II, § 16, p. 45.

41. Cet abandon est une évidence pour Vattel. Cf. Ibid., t. 2, III, 1, § 4, p. 2 : « La nature ne donnant aux hommes le droit d'user de leur force que quand il leur devient nécessaire pour leur défense et pour la conservation de leurs droits [...], il est aisé d'en conclure, que depuis l'établissement des sociétés politiques, un droit si dangereux dans son exercice n'appartient plus aux particuliers $[\ldots] »$.

42. Ibid., t. 1, I, IV, § 38, p. 57.

43. Ibid.

44. Vattel en subit directement les effets lorsque la Prusse envahit la Saxe. 
la souveraineté à l'autonomie de la personne du souverain au sein de la société - unité de l'autorité par concentration des prérogatives comme chez Hobbes -, mais il la caractérise comme indépendance de la nation par rapport aux autres États, c'est-à-dire comme liberté de l'autorité publique dans le choix des moyens pour réaliser la fin de la nation ${ }^{45}$. À ce propos, Haggenmacher fait remarquer qu'en caractérisant la nation souveraine comme libre et indépendante, Vattel lui applique les traits de l'homme à l'état de nature hobbesien, seul juge des moyens de sa conservation. Et ce qui était un fait chez Hobbes devient chez Vattel une qualité normative ${ }^{46}$ : les nations doivent être laissées libres et indépendantes.

Ainsi, le corps politique est achevé avant même la désignation d'un chef puisqu'il est souverain dès qu'il est autonome ${ }^{47}$. Et la nation trouve son unité en elle-même, nul besoin d'un représentant, car elle est «une personne morale » ayant « un entendement et une volonté, dont elle fait usage pour la conduite de ses affaires et [...] elle est capable d'obligations et de droits $»^{48}$.

Si elle le veut, la nation peut cependant « conférer l'autorité suprême ${ }^{49}$ en se choisissant un conducteur. Mais alors que la nation est fondée par un abandon, - chacun cède ses droits -, la souveraineté n'est pas abandonnée mais «confiée » ${ }^{50}$. Cette délégation n'a aucune nécessité, elle n'a de raison que son utilité pratique, la nation pouvant retenir à elle l'autorité publique ${ }^{51}$. Ainsi, si elle installe un chef, l'autorité de ce dernier est «établie ${ }^{52}$, donc subordonnée à l'acte qui la fait naître ${ }^{53}$. Vattel insiste, la souveraineté n'est

45. Et c'est à partir de cette indépendance inter-étatique qu'il détermine les puissances de la souveraineté (puissance de commander, puissance législative, soin des affaires religieuses, etc.), puissances sur lesquelles il ne s'appesantit pas.

46. P. HAGgenMACHER, «Le modèle de Vattel et la discipline du droit international », op. cit., p. 33-34. Vattel défend l'indépendance des nations dès la préface de son ouvrage, affirmation qu'il faut nuancer par les devoirs qui lient les unes les autres. Cf. Ibid., préface, p. 15.

47. Emmanuelle Jouannet résume ce point ainsi : le peuple est à la fois «le détenteur originaire mais également le détenteur définitif du pouvoir souverain, dont il ne fait que déléguer l'exercice à l'organe dirigeant ». Cf. E. JOUANNET, Emer de Vattel et l'émergence doctrinale du droit international classique, op. cit., p. 323.

48. E. de VATTEL, Le Droit des gens, op. cit., t. 1, I, IV, § 40, p. 59-60.

49. Ibid., t. 1, I, V, §56, p. 74. L'élection d'un souverain n'est qu'un moyen parmi d'autres pour réaliser sa fin.

50. Ibid., t. 1, I, IV, §38-39. Nous soulignons. L'auteur utilise également le verbe « conférer », cf. Ibid., t. 1, I, IV, § 40, p. 60 : «Lors donc qu'elle [la société politique] confère la souveraineté à quelqu'un [...]». Nous soulignons. On voit ici la reprise d'une position lockéenne, cf. J. LOCKE, Le Second traité du gouvernement, XIII, Paris, PUF, 1994.

51. Elle fonde alors un gouvernement populaire.

52. Ibid., t. 1, I, IV, § 39, p. 58.

53. Emmanuelle Jouannet explique cette position par l'influence des monarchomaques et de Locke sur Vattel, et apporte pour preuve les références de Vattel à ces auteurs dans l'édition du Droit des gens de 1830, E. Vattel, Le Droit des gens..., Paris, Souza, Laemmert et Cie, 1830 : I, IV, § 45, p. 79-80; §50, p. 83-84; §51, p. 85. Cf. E. JouANNET, «Vattel et la sujétion directe de l'État au droit international » in Simone GOYARD-FABRE (éd.), L'État moderne : regards sur la pensée politique de l'Europe occidentale entre 1715 et 1848, Paris, Vrin, 2000, note 1, p. 160. 
pas une propriété ou un patrimoine ${ }^{54}$. Et le souverain demeure soumis au mandat dicté par la nation qui est de réaliser «le bien commun de tous les citoyens », car, rappelle Vattel, les hommes n'entrent dans la société civile que « pour leur propre avantage et leur salut ${ }^{55}$.

Et même si la nation décide d'élire un souverain, «le droit de se gouverner [lui] appartient toujours essentiellement ${ }^{56}$, et ainsi le souverain, simple « conducteur de la société ${ }^{57}$, devra constamment avoir à l'esprit que l'exercice de la souveraineté ne lui a été remis que pour aider la nation à atteindre sa fin, et il ne doit jamais considérer «le peuple comme un troupeau de bétail dont il doit tirer ses richesses, et duquel il peut disposer pour remplir ses vues et satisfaire ses passions $»^{58}$.

L'absence d'indication sur la dimension contractuelle de la passation de la souveraineté - les termes d'engagement et de pacte ne sont utilisés que pour rendre compte de l'association entre les particuliers - traduit une volonté de ne pas enfermer les sujets dans un pacte qui les conduiraient à leur ruine si le prince se fait tyran. Ainsi, cette délégation non contractualisée de la souveraineté réduit l'autonomie de son bénéficiaire ${ }^{59}$, alors que la nation, à l'inverse, a une consistance ontologique propre.

\section{Deuxième partie - La guerre civile : démembrement ou reconfiguration de la souveraineté}

\section{Conditions de possibilité de la dissension}

\section{a. La part inaliénable du droit naturel}

Si avec Hobbes, le souverain agit au nom de ses sujets, il s'ensuit qu'il est incapable de commettre des injustices envers eux. En effet, au fondement de la République réside un contrat par lequel les particuliers abandonnent leur droit à toute chose, abandon qui ne transfère aucun droit au souverain

54. E. de VATTEL, Le Droit des gens, op. cit., t. 1, I, IV, § 39, p. 58-59. Sur la critique vattelienne des États patrimoniaux et ses conséquences sur la théorie de l'État moderne, voir E. JOUANNET, «Vattel et la sujétion directe de l'État au droit international », op. cit., p. 153-179.

55. E. de VATTEL, Le Droit des gens, op. cit., t. 1, I, IV, § 39, p. 58.

56. Ibid., t. 1, I, V, § 61, p. 76-77.

57. Ibid., t. 1, I, IV, § 39, p. 58. Le terme «conducteur» apparaît à de nombreuses reprises dans l'ouvrage.

58. Ibid., t. 1, I, IV, § 39, p. 59.

59. Vattel insiste sur les devoirs du souverain. Bien qu'il soit le dépositaire des obligations et des droits de la nation, et donc seul juge des moyens de réaliser la fin de la société civile, il doit cependant agir en «père tendre et sage, en fidèle administrateur, veiller pour la nation, prendre soin de la conserver, de la rendre plus parfaite, d'améliorer son état, et de la garantir autant qu'il le pourra de tout ce qui menacerait sa sûreté ou son bonheur ». Ibid., t. 1, I, IV, $\S 41$, p. 60 . 
puisqu'il dispose déjà d'un droit sur tout. Le souverain continue donc d'être libre de tout faire. Ainsi, celui qui l'accuserait d'agir injustement lui reprocherait d'effectuer une action qu'il a autorisée. Le principe est implacable : «celui qui fait quelque chose parce qu'un autre lui a donné le pouvoir de le faire ne commet aucune injustice envers celui-ci en agissant ${ }^{60}$. Toute opposition d'un sujet à l'autorité du chef de l'État contredit l'acte volontaire par lequel il s'est fait son obligé. Blâmer le souverain revient en fait à s'accuser soi-même.

Cette position s'appuie sur le concept de justice proposé par Hobbes qui ramène le juste à ce qui n'est pas empêché par une loi civile, et l'injuste à la transgression d'une convention passée lorsqu'il existe un pouvoir capable de contraindre les contractants à s'exécuter ${ }^{61}$. Raison pour laquelle la justice et l'injustice n'ont d'existence que relativement à la présence d'une société. Et ainsi, il n'y a de sens à parler de juste que s'il existe des lois civiles, donc un souverain, c'est-à-dire des conventions ${ }^{62}$. Il est donc incohérent de reprocher au souverain d'avoir commis une injustice, car, n'ayant contracté avec nul autre, il ne peut rompre de convention.

Par conséquent, après l'institution du souverain, toute action d'un sujet reposant sur une volonté non conforme à celle par laquelle il a fait le souverain, est une contradiction. Toute rupture du pacte revient donc, pour un sujet, à nier ce qu'il veut. De la sorte, Hobbes paraît avoir sapé toute possibilité de fonder en droit une opposition du sujet à son souverain.

Mais bien que le souverain puisse tout entreprendre, et même attaquer des sujets lorsque cela lui paraît nécessaire au maintien de la paix, si ceux-ci ne disposaient d'aucune ressource à opposer à ses actions préjudiciables, la fondation de la société civile leur serait inutile ${ }^{63}$. Quel bénéfice retireraientils de l'abandon de leur liberté naturelle s'ils étaient aussi vulnérables dans l'état civil que dans l'état de nature ${ }^{64}$ Hobbes répond à ce problème : la désobéissance d'un sujet n'est pas injuste lorsque le souverain s'en prend à son intégrité physique.

60. T. HobBES, Léviathan, op. cit., II, XVIII, p. 295.

61. Ibid., II, XXVI, p. 406.

62. Cette transformation a pour effet de faire de la loi une production toute immanente : elle est entièrement humaine, et non plus théologique, et elle est toute contenue dans les décisions du souverain.

63. Pour autant, Hobbes ne considère pas que l'individu puisse rompre le pacte dès qu'il a le sentiment de ne plus être en sécurité. Le pacte social n'a pas qu'une finalité individuelle et égoïste, sinon, la société serait composée par des freeriders qui seraient en droit de la quitter dès qu'ils n'y trouveraient plus leur compte. L'abandon de sa liberté naturelle est un acte politique en ce qu'il permet la paix de tous. Sur la question de l'intérêt du sujet à se soumettre au souverain et sur ce qui est compris dans l'acte d'autoriser voir D. GAUTHIER, «Symposium Papers, Comments and an Abstract: Hobbes's Social Contract », in Noûs, n 1, vol. 22, 1988, p. $71-82$, p. 77.

64. Il serait plus encore contradictoire d'affirmer que la constitution de l'État est un acte libre par lequel le sujet, calculant les conditions de sa conservation, accepte d'abandonner son droit naturel y compris s'il ne bénéficie pas de la sécurité qui justifiait cet abandon. 
La possibilité de cette désobéissance se comprend grâce au modèle hobbesien de la convention. Toute convention, c'est-à-dire tout accord volontairement passé pour restreindre sa liberté, ne peut avoir pour fin que l'obtention d'un bien, car la volonté ne peut vouloir que ce qu'elle se figure comme tel. Un individu ne peut donc s'engager à se nuire, et certains droits ne peuvent faire l'objet d'une convention et être abandonnés. C'est le cas pour « le droit de résister à ceux qui vous agressent par la violence pour vous ôter la vie ${ }^{65}$, et ce y compris s'il s'agit du souverain. Ainsi, une convention par laquelle un individu s'engage à ne pas empêcher un autre de le blesser est nulle puisqu'elle serait une contradiction de la volontét ${ }^{66}$.

Par conséquent, lorsque le souverain ordonne à un sujet de se nuire, il effectue une action pour laquelle il n'a reçu aucune autorisation. De ce fait, le sujet récupère la part inaliénable de sa liberté naturelle qu'il n'a pu s'engager à abandonner. Cette liberté que Hobbes nomme « vraie liberté » ${ }^{67}$ [true liberty] des sujets - vraie car non circonscrite par la volonté du souverain, résidu de la liberté naturelle au sein de l'état civil - permet de désobéir $^{68}$. Elle justifie même que le sujet défende son corps au cas où le souverain l'attaque, sans qu'on ne puisse lui reprocher de rompre le pacte social $^{69}$.

Mais le fait que le sujet conserve un moyen de se protéger n'implique pas qu'il en ait, à strictement parler, le droit. En effet, la désobéissance et la résistance d'un sujet ne peuvent être dites justes - le juste étant défini par le respect des conventions, or entre le sujet et le souverain il n'y a aucun contrat -, elles sont non injustes ${ }^{70}$. En effet, le sujet mis en danger par son souverain est plongé dans l'état de nature, raison pour laquelle, en refusant d'obéir ou en se défendant grâce à son droit naturel, il mobilise un droit qui n'est pas civil. Il agit en vertu d'un droit extra légal, issu de l'état de nature, sur lequel le souverain n'a aucune prise. Et même si le souverain a parfaitement le droit de s'en prendre à lui et ne commet aucune injustice en agissant ainsi, il permet de ce fait à sa victime de se soustraire sans injustice à son autorité ${ }^{71}$.

65. T. HobBes, Léviathan, I, XIV, p. 234.

66. Ibid., I, XIV, p. 235.

67. Ibid., II, XXI, p. 345.

68. Martine Pécharman fait remarquer que cette résistance n'invalide pas le droit de punir du souverain: "L'exercice par le souverain de son pouvoir de punition n'est nullement incompatible avec la résistance du condamné à l'application de la sentence. Qu'il soit ou non coupable n'entre pas en jeu ici : le condamné, même s'il accepte sa condamnation comme juste, est toujours en droit de résister à la force qui essaie de s'emparer de lui, de l'entraver, de l'emprisonner, de le supplicier, de le mettre à mort». Cf. M. PECHARMAN, « Hobbes» in Michela MarZano (dir.), Dictionnaire de la violence, Paris, France, Presses universitaires de France, DL 2011, p. 629-630.

69. Hobbes le démontre par l'analyse des paroles expresses.

70. L'expression de Hobbes est « refuser de faire sans commettre une injustice », «without injustice refuse to do », Léviathan, op. cit., II, XXI, p. 347-348.

71. Cf. G. LeBrun, « Hobbes en deçà du libéralisme », in Manuscrito, Revue de l'Université de Campinas, Brésil, vol. IV, n 1, 1980-1981, p. 37-49. 


\section{b. Limites à la puissance du souverain}

Pour le diplomate suisse, les réquisitoires contre le souverain n'ont rien d'une contradiction des sujets avec eux-mêmes, car la puissance du souverain n'est pas absolue. En effet, immédiatement après avoir indiqué que l'élection d'un souverain emporte avec elle l'obligation de lui obéir, Vattel ajoute que «cette obéissance ne doit pas être absolument aveugle ${ }^{72}$. Bien que la soumission au souverain soit nécessaire pour qu'il puisse veiller au salut de la nation, et que, par conséquent, les sujets doivent toujours supposer que ses 《ordres sont justes et salutaires ${ }^{73}$, cette soumission n'est pas absolue.

La puissance du souverain est restreinte par plusieurs limites, dont, en premier lieu, la finalité de l'association politique (justice, sécurité, propriété, bonheur) et la loi naturelle. Cette dernière consiste en un ensemble de normes que la raison tire de l'adéquation entre des actions et la nature de $l^{\prime}$ homme ${ }^{74}$. Or, nous dit Vattel, toute action du souverain contraire à ces règles est effectuée «sans droit véritable ${ }^{75}$. $\mathrm{Si}$, par exemple, il contraint un sujet à agir contre la loi naturelle, fondement même du droit, ou s'il ruine la sûreté de ce dernier - ce qui revient à faire disparaître l'utilité de la société civile pour lui -, alors l'obligation que le sujet a d'obéir au souverain disparaît.

Les réactions face au souverain dépendent de la nature de la violence et de son extension. Si les attaques ne frappent qu'un individu, il ne peut que désobéir, pour ne pas manquer à la loi naturelle, et faire preuve de patience. Cette recommandation, qui parcourt la philosophie politique depuis Tertullien $^{76}$, en passant par Augustin ${ }^{77}$ et Thomas $^{78}$ n'a, à l'Âge classique, plus rien de l'attente religieuse d'une punition divine des tyrans. Elle est plutôt une incitation à maintenir l'ordre en attendant que le souverain fasse un usage plus réglé de son droit de punir ${ }^{79}$. La priorité va à la protection de

72. E. de VATTEL, Le Droit des gens, op. cit., t. 1, I, IV, § 54, p. 70.

73. Ibid., § 53, p. 70.

74. E. de VATTEL, La Loi naturelle peut-elle porter la société politique à la perfection sans le secours des lois politiques?, Question de l'Académie de Dijon de 1742, in Le Droit des gens ou principes de la loi naturelle appliqués à la conduite et aux affaires des nations et des souverains, traduit par Paul Pradier-Fodéré, Libr. de Guillaumin et Cie., 1863, t. 1, § 6, p. 36 : «La loi naturelle consiste dans les règles de conduite, fondées sur la nature des choses, et particulièrement sur la nature de l'homme. Nous la connaissons par la raison. Cette faculté de l'âme nous découvre la convenance ou la disconvenance qui se trouve entre les actions libres et la nature des choses et de l'homme en particulier».

75. E. de VATTEL, Le Droit des gens, op. cit., t. 1, I, IV, § 54, p. 71.

76. Tertullien, De la patience, traduit par Jean-Claude Fredouille, Paris, France, Éd. du Cerf, 1999, XIII.

77. Augustin, De la Patience, in Euvres complètes, traduit par Jean-Baptiste Raulx, Bar-le-Duc, France, L. Guérin, 1864, tome XII, p. 294-305.

78. Cf. Thomas d'AQuin, Du gouvernement royal, traduit par Claudet Roguet, Paris, France, Éditions Saint-Rémi, 2008, I, VI.

79. Voir l'exemple de Charles VI donné par l'auteur, cf. E. de VAtTEl, Le Droit des gens, op. cit., t. 1, I, IV, $\S 54$, p. 73. 
l'ordre politique contre l'intérêt du sujet, car ce serait "retomber dans l'état de nature et rendre le gouvernement impossible ${ }^{80}$ que de soumettre le souverain à l'appréciation des particuliers.

Cependant, cette patience ne s'applique qu'à deux formes d'injustices, les «douteuses » et « supportables » ${ }^{81}$, et non à celles qui sont «manifestes et atroces $»^{82}$. La distinction est de Barbeyrac qui, dans sa traduction du De jure belli, faisait valoir contre Grotius que l'obligation que les sujets ont de se plier aux injustices de leur prince ne concerne que celles qui sont imputables à la faiblesse de sa nature ; tout homme abuse parfois de son pouvoir. Et lorsqu'il s'agit d' « injustices manifestes et insupportables », les sujets ne doivent les endurer que si leur résistance cause des maux plus terribles pour la société civile que ceux dont le prince est responsable par ses abus ${ }^{83}$. Par cette distinction Barbeyrac prétendait empêcher autant la guerre civile que la tyrannie ${ }^{84}$. Puisqu'il est dans l'intérêt des sujets eux-mêmes qu'ils n'aient pas le droit de ruiner l'autorité de leur souverain à chaque injustice - sinon il serait incapable de réaliser la fin de la société politique -, la solution de Barbeyrac est de n'admettre la résistance que si la violence est montée aux extrêmes ${ }^{85}$.

\section{Ibid., p. 71. Nous soulignons.}

81. Ibid. : «Un sujet doit souffrir avec patience, de la part du prince, les injustices douteuses et les injustices supportables : les premières, par la raison que quiconque s'est soumis à un juge, ne peut plus juger lui-même de ses prétentions ; les injustices supportables doivent être sacrifiées à la paix et au salut de l'État, en faveur des avantages que l'on retire de la société ». Sur le thème de la patience, une citation de Tacite est constamment reprise par les auteurs de l'École du droit naturel et des gens. Cf. TACITE, Euvres complètes. Les histoires, IV, LXXIV, traduit par C.-P. Salles, Paris, R. Laffont, 2014, p. 392 : «On supporte une récolte manquée, les pluies excessives et les autres fléaux de la nature : supportez de même les dérèglements ou l'avarice des tyrans. Il y aura des vices tant qu'il y aura des hommes, mais ce mal n'est pas continuel et entre-temps il est compensé par l'avènement du bien ».

82. E. de VATTEL, Le Droit des gens, op. cit., t. 1, I, IV, §54, p. 71. Historiquement, le vocabulaire du manifeste a été employé dans le droit pour désigner les crimes ne nécessitant pas d'instruction judiciaire. Chez les auteurs du droit des gens, ce vocabulaire est régulièrement associé à l'idée d'injustice pour rendre compte des actes du souverain qui déborde de son droit de punir. Cf. Hugo Grotius, Le Droit de la guerre et de la paix, traduit par Jean Barbeyrac, E. Thourneisen, 1746, II, XX, $\S 43.3$ : «crimes très atroces et manifestes »; II, XXV, § 8.2: «oppression manifeste »; S. von PUFENDORF, Le droit de la nature et des gens, op. cit., VIII, VI, § 14 : «injures manifestes »; J.-J. BuRLAMAQUI, Principes du droit de la nature et des gens, Paris, B. Warée, Librairie de la Cour royale, 1820, t. $4,2^{\mathrm{e}}$ part., ch. VI, $\S 8$, p. 272 : « injures manifestes ».

83. Cf. Hugo Grotius, Le Droit de la guerre et de la paix, op. cit., I, IV, § 2, note 1, p. 171.

84. Ibid., préface du traducteur, p. XXXIX : «... des principes de politique sur lesquels je raisonne ici et ailleurs, également éloignés de favoriser l'esprit de sédition et d'ériger en droit la licence et du despotisme».

85. Derathé note l'influence de Locke sur Barbeyrac, influence qui se fait également sentir sur Vattel lui-même. Cf. J. LOCKE, Le Second traité du gouvernement, Paris, PUF, 1994, XIX, $\S 239$. Si le roi manque la finalité du gouvernement civil (le bien public et la protection de la propriété privée), il se met en état guerre avec son peuple. Voir R. D ERATHE, Jean-Jacques Rousseau et la science politique de son temps, Paris, J. Vrin, 1970, p. 91, note 1. 
Contrairement à Barbeyrac, Vattel distingue les injustices « douteuses », que le sujet, privé de son droit de sûreté, n'est plus en mesure d'évaluer, et les «supportables » dont l'endurance est inférieure aux avantages tirés de la stabilité de l'État ${ }^{86}$. Ainsi, l'évaluation des effets de la résistance sur le bien public, que Barbeyrac utilisait pour restreindre le recours à la résistance en cas d'injures manifestes, sert chez Vattel à justifier que le peuple endure les injustices supportables. Vattel radicalise donc la position de Barbeyrac puisqu'il défend que les sujets ont le droit de résister à leur souverain dès que ses injures sont «manifestes et atroces", sans avoir à envisager les conséquences de leur geste sur l'ordre politique ${ }^{87}$. Lorsque par caprice un prince attaque la vie ou les biens d'un sujet ${ }^{88}$, sans que cela ne l'aide à réaliser la fin de la société politique, alors il n'est plus permis de douteur de la nature de sa violence, et les sujets peuvent entraver son action.

Deux arguments viennent appuyer cette thèse. Un sujet peut s'opposer à son souverain lorsqu'il s'en prend sans raison à sa vie ou à ses biens, car l'obligation naturelle de veiller à sa sûreté ne peut être «entièrement et absolument ${ }^{89}$ aliénée. L'argument rappelle Hobbes : il est impossible d'abandonner le souci de sa préservation. Mais Vattel, moins attaché à la dimension contractuelle du pacte d'institution du souverain, insiste moins sur l'impossibilité d'une convention contraire au bien du contractant que sur l'incohérence qu'il y aurait, pour les sujets, à devenir membre d'une société sans en retirer un avantage pour leur sûreté. En outre, le sujet peut résister, car l'institution du souverain, toute contingente, lui attribue une sacralité relative, c'est-à-dire subordonnée à ses actions ${ }^{90}$. En manquant à la finalité de son investiture, le souverain perd la majesté de son statut, et agissant sans droit - ce qui est un non-sens pour Hobbes -, il devient un « ennemi injuste $»^{91}$.

Une restriction nuance cependant ce point. Tant que le souverain n'offense qu'un seul sujet, ce dernier ne peut recourir qu'à une violence défensive. Par égard à l'utilité du souverain pour la nation, il est préférable de fuir plutôt que de menacer un ordre bénéfique aux autres sujets ${ }^{92}$. Et ainsi,

86. Étrangement Vattel semble ici identifier la présence du souverain avec l'existence de la société ou plutôt, faire du souverain la condition de possibilité de l'État.

87. Vattel reprend presque mot pour mot Burlamaqui, cf. J. BURLAMAQUI, ibid.

88. E. de VATTEL, Le Droit des gens, op. cit., t. 1, I, IV, §54, p. 72 . Vattel illustre les «injures manifestes et atroces» par les cas où le prince viole toutes les règles, refuse la mesure, ou s'en prend à la vie d'un innocent.

89. Ibid.

90. Contrairement à Hobbes, qui n'admet la résistance qu'en cas d'attaque contre l'intégrité du sujet, Vattel distingue entre des finalités politiques et non politiques de la punition du prince, ce qui est inconcevable dans la perspective hobbesienne.

91. Ibid.

92. Vattel donne les exemples de David fuyant la hargne de Saül, et de la garde rapprochée de Charles VI qui, devant son premier accès de folie meurtrier, le mit hors d'état de nuire au lieu de le tuer. Ibid., § 54, p. 73. 
lorsque les injustices sont attestées, Vattel tranche en faveur de l'autorité du souverain et sacrifie le bonheur de l'individu.

Seule la nation est en droit de s'en prendre à son souverain. Dans le cas où la souveraineté transmise au souverain est limitée par une constitution, et que ce dernier l'ignore alors qu'il doit s'y soumettre, la nation est libérée du lien d'obligation juridique. De ce fait, elle peut révoquer celui qu'elle a mandaté. Et même si la souveraineté est transférée sans borne, c'est nécessairement avec «la réserve tacite ${ }^{93}$ que l'autorité publique soit employée pour réaliser la fin de la société civile et non pour la détruire. Ainsi, le peuple attaqué par son souverain est dans une situation dont le caractère extrême justifie qu'il puisse le renverser.

\section{Sens de la dissension}

\section{a. Vanité et monstruosité}

Bien que Hobbes admette la non-injustice de la résistance, elle ne constitue pas un droit. C'est pourquoi son statut ne peut servir à fonder une opposition formelle et collective au souverain. D'où le traitement qu'il réserve à la dissension lorsqu'elle s'intensifie et prend la forme d'une guerre civile.

Contraire au mouvement d'institution du politique produit par un calcul de la raison, cette guerre est si, on se rapporte à la genèse de l'État, un retour à l'état de nature. Elle est la résurgence, au sein du politique, de ce passé fictif que les contractants ont cherché à fuir en suivant les lois de nature ${ }^{94}$. Cette guerre est ainsi contradictoire en ce qu'elle fait renaitre la disposition à l'affrontement de chacun contre chacun alors même que les conditions de la paix ont été satisfaites. Cette incohérence est mise en évidence par Hobbes à travers le vocabulaire de la maladie et de la monstruosité qui lui sert à décrire l'État qui en est victime.

Ce vocabulaire se justifie par le fait que cette guerre déploie à l'extrême une dissension à laquelle Hobbes refuse de faire place puisque les propriétés de la souveraineté - droits dont le souverain est seul à disposer - ne peuvent être séparées de sa personne et divisées ${ }^{95}$. L'idée que l'opposition entre les voix et les idées soit le moteur d'un progrès est radicalement absente de la

\footnotetext{
93. Ibid., § 51, p. 66.
}

94. Sur les difficultés liées au recours à la guerre civile pour penser l'état de nature, voir N. GRANGE, «La cité en guerre. Violence naturelle ou violence politique ? Un aspect de la guerre civile chez Hobbes », op. cit., p. 17-38.

95. L'exercice de la souveraineté, qu'il soit le privilège d'un individu ou de plusieurs unis en une personne, n'altère pas l'indivisibilité de la puissance la plus grande qui soit. De même, les différences entre régimes, relatives au nombre d'individus composant la personne du souverain, ne changent rien à l'exercice du pouvoir. L'indivisibilité de la souveraineté est défendue contre les parlementaires Anglais pour lesquels le pouvoir royal ne doit pas être absolu mais limité par le Parlement. 
pensée de Hobbes, en témoigne sa considération pour le gouvernement mixte :

À quelle maladie du corps humain naturel peut être correctement comparée cette déformation de l'État, je ne sais. Mais j'ai vu un homme qui en avait un autre qui lui poussait sur le côté, avec une tête, des bras, une poitrine et un ventre ; s'il en avait eu un autre, lui poussant sur l'autre côté, la comparaison aurait alors été correcte ${ }^{96}$.

Ce gouvernement dissocie les fonctions du corps politique en en distribuant l'exercice à des personnes distinctes et a donc plusieurs âmes. Hobbes illustre son propos: lorsque la puissance de lever des impôts, fonction nutritive, dépend d'une personne, que la puissance de diriger, faculté motrice, dépend d'une autre et la puissance législative, faculté rationnelle, d'une troisième qui doit s'accorder avec les deux premières ${ }^{97}$, le corps est déficient. Ce n'est donc pas un État mais trois factions indépendantes. De la division de l'autorité s'ensuit donc une perte de puissance et un affaiblissement de la souveraineté, chacun des organes prétendant représenter le tout du peuple, peuple qui n'est un que par l'unité de son représentant ${ }^{98}$.

On comprend alors que la guerre civile, conflit où le corps politique dirige sa violence contre lui-même, soit traitée comme une déformation de l'État. Si, à la suite d'Aristote, le monstrueux désigne l'être dont la matière a résisté au processus d'information censé lui donner sa nature ${ }^{99}$, la guerre civile est une guerre de mauvaise forme. La seule bonne guerre est celle que les États se livrent, car incapables d'instituer une puissance supérieure à même de les contraindre, ils demeurent dans l'état de nature ${ }^{100}$.

96. T. HobBES, Léviathan, op. cit., II, XIX, p. 489.

97. Ibid., II, XIX, p. 488. Dans un tel corps politique, la concorde entre les institutions est bien trop hasardeuse. Si les impôts ne sont pas remis au souverain, alors l'accès à la nourriture du corps politique est soumis à la volonté d'un autre, mettant en danger la possibilité même d'une armée forte en cas de besoin de se protéger, impliquant qu'il soit impossible pour le souverain de faire suivre sa volonté par ses sujets.

98. Sur ce point voir J. TERREL, «Hobbes : la critique du gouvernement mixte », in Marie GAILlE-NiKODimOV (éd.), Le Gouvernement mixte: de l'idéal politique au monstre constitutionnel en Europe, XIIIe-XVIIe siècle, Saint-Étienne, France, Publications de l'Université de Saint-Étienne, 2005, p. 175-189.

99. ARistote, De la génération des animaux, traduit par Pierre Louis, Paris, France, les Belles Lettres, 2002, IV, 3-4, et Physique, traduit par Pierre Pellegrin, Paris, France, GFFlammarion, 2000, II-8.

100. Le droit des gens n'est pour Hobbes que l'application de la loi naturelle entre les souverains. Cf. Du Citoyen, op. cit., II, XIV, § 4 ; Léviathan, op. cit., II, XXX, p. 518. Hobbes fait par ailleurs remarquer que les souverains souffrent bien moins de l'état de nature que les particuliers. Pour une position plus nuancée sur l'état de nature entre souverains chez Hobbes, voir R. TUCK, "Grotius, Hobbes and Pufendorf on humanitarian intervention » in Stefano RECCHIA et Jennifer M. WELSH (éds.), Just and unjust military intervention: European thinkersfrom Vitoria to Mill, New York, Cambridge University Press, 2013, p. 107-110. 
Le caractère contre-politique de cette guerre s'atteste par son origine. Elle naît de la passion de vanité qui pousse les sujets à désirer une reconnaissance supérieure à celle dont ils font l'objet. Sous l'effet de la vanité, chacun veut que son opinion soit adoptée par le groupe et cherche ainsi à se distinguer ${ }^{101}$. Or ce désir d'être honoré plus que les autres brise l'égalité de condition des sujets vis-à-vis du souverain. Incapables de percevoir leur juste valeur, les hommes en viennent à ne plus se reconnaître comme semblables, fragilisant ainsi la concorde interne.

De plus, pour satisfaire ce désir de reconnaissance, chacun est incité à créer par lui-même un pouvoir politique, voire à s'approprier celui dont le souverain est détenteur. Ainsi, le cœur de la guerre civile est la tentative de rejouer ce moment inaugural du pacte par lequel la puissance la plus grande qui soit fut produite. En effet, la faction [faction] est l'unité agissante de la guerre civile, constituée par la création secrète d'une puissance cachée grâce à des «pactes conclus mutuellement [...] sans l'autorité de celui ou ceux qui détiennent la souveraineté ${ }^{102}$. Or, cette création de pouvoir, une fois la société civile établie, est illégale.

En effet, toute convention par laquelle des individus se promettent une défense mutuelle une fois le souverain institué - c'est-à-dire une fois que chacun est en sécurité - contredit le pacte social. Une telle convention suppose de se dédire de l'engagement par lequel la paix interne a pu être instaurée, ce qui met en péril l'autorité du souverain. Alors qu'elle serait licite dans l'état de nature, dans l'état civil elle est entièrement frauduleuse :

car tout de même que la première union des hommes les a tirés de l'état de nature pour les ranger sous le gouvernement d'une police, la faction les soustrait à celle-ci par une nouvelle union des sujets entièrement irrégulière ${ }^{103}$.

Non seulement illégale, la sédition est irrationnelle car contraire à la loi naturelle. Le souverain disposant de fait de la puissance la plus grande qui soit, son existence assure la paix et suffit à donner pleine satisfaction aux séditieux, raison pour laquelle la guerre civile ne peut avoir aucune motivation politique.

En outre, l'effet de la sédition sur la concorde est désastreux. La sédition ne peut demeurer une connivence au sein d'un groupe limité d'individus, elle doit s'étendre pour survivre. En effet, les séditieux passent une convention illégale par laquelle ils créent un pouvoir qui échappe à l'autorité du souverain. Mais en répétant l'union fondatrice par laquelle l'état de

101. Sur l'effet du désir de gloire sur le corps politique, et le rôle des passions dans l'avènement de la guerre civile voir Julie SAADA-GENDRON, «L'analyse des passions dans la dissolution du corps politique : Spinoza et Hobbes », in Astérion. Philosophie, histoire des idées, pensée politique, $\mathrm{n}^{\circ}$ 3, 9 septembre 2005.

102. T. HobBes, Du Citoyen, op. cit., II, XIII, § 13, p. 264.

103. T. HobBes, Le Citoyen ou les Fondements de la politique, traduit par Samuel Sorbière, Paris, Flammarion, « Garnier-Flammarion », 1982, II, XIII, § 13, p. 236. Nous choisissons ici la traduction de Sorbière, car elle insiste sur l'irrégularité de cette union. 
nature a pu être quitté ${ }^{104}$, la sédition réitère un acte déjà effectué et absolu en ce qu'il engage pour toujours. Et la puissance créée par les séditieux menace celle du souverain donc la paix civile dont jouissent tous les citoyens. Par conséquent, l'effet de cette convention secrète est de faire réapparaître l'état de nature au sein de l'état civil. Il est logique dès lors que les factieux soient forcés, pour survivre, de contraindre les autres à reconnaître le pouvoir qu'ils ont généré, c'est-à-dire à s'y soumettre ou à périr, reproduisant ainsi, dans l'État, le mécanisme d'assujettissement et d'élimination qui prévalait à l'état de nature. Ainsi, en plus d'être contraire au droit, la faction, qui est « comme un État dans un État ${ }^{105}$, est source d'une violence qui ne peut que s'étendre à l'ensemble de la société.

Cette comparaison entre la faction et un État naissant au sein d'un autre laisse penser qu'elle puisse devenir, une fois son développement achevé, un corps politique stable. Hobbes en récuse la possibilité par le concept de faction. À son fondement réside une promesse qui ne peut être garantie par une puissance suffisamment grande pour contraindre les factieux contractants à s'exécuter. Par conséquent, la faction n'est qu'une parole donnée qui peut à chaque moment être reprise. Ainsi, malgré la connivence qui les unit ponctuellement, les factieux demeurent à l'état de multitude et sont entre eux à l'état de nature ${ }^{106}$. La faction ne peut donc que se dissoudre en individus atomisés.

Ainsi, si l'État est un corps au sein duquel la souveraineté est une «âme artificielle » dont la fonction est de protéger le peuple, si la souveraineté est ce principe de vie et d'unité qui donne au corps son identité et sa bonne forme, alors, tranche Hobbes, « la concorde est sa santé, la sédition est sa maladie, et la guerre civile est sa mort ${ }^{107}$.

Dès lors, le souverain ne doit pas lutter contre les séditieux par des sanctions pénales. Puisque les rebelles se comportent envers le souverain et les autres sujets comme s'ils étaient toujours dans l'état de nature ${ }^{108}$, ils doivent être sanctionnés par la guerre. Cette punition est en fait un recours au droit naturel, et elle peut être effectuée par le souverain comme par les particuliers. Puisqu'ils fragilisent la sécurité de tous, alors chaque sujet doit recourir à son droit naturel contre les factieux et est en droit de les mettre à mort. Cette guerre, que chacun doit leur mener au nom de la loi de nature, ne leur attribue aucune reconnaissance ou souveraineté. Elle est une résurgence

104. L'association n'est une rébellion, et donc n'est illicite, que du fait de sa temporalité (post-contrat social).

105. T. HobBes, Du Citoyen, op. cit., II, XIII, § 13, p. 264.

106. T. HobBes, Éléments, op. cit., II, I, § 2, p. 227.

107. T. HobBes, Léviathan, op. cit., p. 64. Pour autant Hobbes ne considère pas que les séditieux visent la destruction de l'État, c'est-à-dire un retour à l'état de nature, mais plutôt une nouvelle répartition du pouvoir. Cf. T. HobBES, Du Citoyen, op. cit., II, VI, $\S 13$, p. 174-175.

108. Sur le problème du «free-rider», voir A. BOYER, Chose promise: étude sur la promesse, à partir de Hobbes et de quelques autres, Paris, Presses universitaires de France, 2014. 
de celle qui anime les particuliers à l'état de nature, c'est-à-dire une guerre d'assujettissement ou d'élimination sans aucun égard ${ }^{109}$.

Ce recours à la guerre contre les séditieux fait ainsi disparaître le péril de la guerre civile pour la stabilité de la République. Or, en se faisant ennemis de l'État par leurs pactes secrets, les rebelles se mettent eux-mêmes à l'extérieur de l'Etat et indiquent qu'ils n'en font plus partie ${ }^{110}$. De la sorte le remède de Hobbes consiste à traiter la guerre interne comme une guerre externe.

\section{b. Une scission féconde}

La position de Vattel ne peut pas être plus éloignée puisqu'il soutient que la guerre civile, dès lors qu'elle surgit, doit être traitée comme l'expression d'une inquiétude légitime des sujets.

On ne prend pas le risque de s'insurger contre son souverain pour se distinguer, comme le prétend Hobbes, mais parce qu'on craint qu'il soit incapable de protéger nos droits soutient le diplomate. La dissension naît de la peur que les sujets peuvent avoir de ne pas obtenir de réparation au cas où ils subiraient des préjudices ${ }^{111}$. D'une certaine manière, le souverain est donc celui qui porte la responsabilité de la guerre civile.

Cependant, et ce quelle que soit sa forme - Vattel divise le phénomène de la contestation en plusieurs unités ${ }^{112}:$ rebelles $^{113}$, émotion populaire ${ }^{114}$, sédition $^{115}$, soulèvement ${ }^{116}$, guerre civile -, la contestation du souverain est

109. T. HobBes, Léviathan, op. cit., II, XXVIII, p. 467. Sur ce point cf. Martine PeCHARMAN, art. « Hobbes », in Michela MARZANO (dir.), Dictionnaire de la violence, op. cit., p. 630.

110. Martin Pécharman parle de «non-sujets » ou d' «anti-citoyens », cf. Ibid.

111. Lorsque le souverain n'agit pas pour que disparaisse la crainte qu'ont les sujets de ne pas voir leurs droits défendus, alors «la confusion, le désordre, le découragement, naissent bientôt dans l'État» et «les vertus civiles s'éteignent, et la société s'affaiblit». Cf. E. de VATTEL, Le Droit des gens, op. cit., t. 1, I, XIII, § 158, p. 154.

112. Ces différents éléments relèvent tous d'une insoumission, que ce soit sous la forme passive d'une désobéissance ou active d'une résistance, selon des intensités et des extensions différentes.

113. Ibid., t. 3, III, XVIII, § 288, p. 198-199: «On appelle rebelles tous les sujets qui prennent injustement les armes contre le conducteur de la société, soit qu'ils prétendent le dépouiller de l'autorité suprême, soit qu'ils se proposent seulement de résister à ses ordres, dans quelque affaire particulière, et de lui imposer des conditions ».

114. Ibid., p. 199: «L'émotion populaire est un concours de peuple, qui s'assemble tumultuairement et n'écoute plus la voix de ses supérieurs, soit qu'il en veuille à ces supérieurs eux-mêmes ou seulement à quelques particuliers. On voit ces mouvements violents, quand le peuple se croit vexé, et nul ordre n'y donne si souvent occasion, que les exacteurs des Impôts ».

115. L'émotion populaire s'appelle sédition lorsqu'elle donne lieu à une «désobéissance formelle ». Ibid.

116. Une différence de degré sépare le soulèvement de la sédition. Il y a soulèvement lorsque la sédition « s'étend, gagne le grand nombre, dans la Ville, ou dans la Province, et se soutient, en sorte que le Souverain même n'est plus obéi ». Ibid. 
globalement condamnée au motif que la paix, comprise comme «ordre public ${ }^{117}$, est condition de la sûreté de chacun.

Mais du fait du prisme par lequel il examine le statut de la dissension, Vattel est empêché de reprendre la condamnation classique de la guerre civile. En effet, la question qui le préoccupe est celle de savoir si un souverain doit suivre les lois de la guerre quand il combat des rebelles. Traitée depuis Cicéron, la question avait chez ce dernier pour réponse que les lois de la guerre ne s'appliquent qu'entre souverains. Les rebelles, ennemis de tous ${ }^{118}$ classés parmi les brigands et les pirates, ne méritent pas une guerre qui suive le droit des gens et leur attribue une reconnaissance. Ainsi contre eux, tout peut être fait.

Cette question est cruciale pour Vattel au point qu'il fait de la réaction du souverain face aux rebelles un indice de sa nature. S'il traite les rebelles comme des citoyens « courageux et fermes, qui exhortent le peuple à se garantir de l'oppression, à maintenir ses droits et ses privilèges » ${ }^{119}$, alors il est un bon prince soucieux du salut de l'État. Si, au contraire, il ne les considère que comme des séditieux épris de dispute, il ne peut être qu'un tyran plein de fureur.

En conséquence, si les rebelles prennent les armes à cause de «justes sujets de plainte ${ }^{120}$, la faute de ces «malheureux ${ }^{121}$ est légère, car, manquant de patience et non de fidélité, ils ont entrepris de se faire justice par eux-mêmes. La punition doit alors être douce. Mais si le soulèvement n'a aucun motif, «ce qui, s'empresse d'ajouter l'auteur, n'arrive peut-être jamais $»^{122}$, alors la punition doit être sévère. Or, en plus de ne pas croire à ce cas de figure, Vattel introduit une restriction : la punition ne peut être dure qu'à la condition que les insurgés soient peu nombreux, car on ne peut dépeupler une ville pour punir son insoumission ${ }^{123}$. L'intérêt du corps social prime donc sur l'exigence de punition.

\section{Ibid.}

118. CiCERON, Les Devoirs, III, 107-108, traduit par Maurice Testard, Paris, Les Belles Lettres, «Collection des universités de France », 1965. Cicéron en fait les ennemis de tous : «communis hostis omnium», Pufendorf en fera les ennemis du genre humain, cf. S. von PUFENDORF, Le droit de la nature et des gens, ou Système général des principes les plus importants de la morale, de la jurisprudence et de la politique, op. cit., VIII, IV, § 5 : « hostis humani generis ».

119. E. de VATTEL, Le Droit des gens, op. cit., t. 3, III, XVIII, § 290, p. 200.

120. Ibid. Vattel ne définit pas ces justes plaintes à cet endroit. Il s'agit sans doute des motifs justes de la résistance qui sont les attaques du souverain contre les biens, la vie des sujets, le manque de réparations en cas d'injustices.

121. Ibid.

122. Ibid., § 291, p. 201. L'idée d'une sédition gratuite n'est pas convaincante pour Vattel.

123. L'auteur ajoute : «La punition la plus juste en elle-même devient cruauté, dès qu'elle s'étend à un trop grand nombre de gens ». Ibid, § 290, p. 200. 
Cette surprenante bienveillance à l'égard des dissidents s'éclaire par le projet général de Vattel ${ }^{124}$. En effet, conseiller privé pour le cabinet de l'électeur de Saxe, Vattel a une expérience directe de la guerre. L'invasion de la Saxe par la Prusse - grâce à une offensive pour lui choquante car non déclarée - force la cour à fuir et marque le début de la Guerre de Sept ans, pendant laquelle il publie Le Droit des gens. Or, une partie des combats contre Frédéric II se déroulant sur son territoire, la Saxe est profondément ravagée par un conflit pour lequel elle n'était pas préparée ${ }^{125}$. L'effort manifesté par Vattel pour défendre un droit des gens nécessaire, irréductible au consentement des souverains, s'y rapporte ${ }^{126}$. En conséquence, ces règles nécessaires, qui s'imposent aux nations malgré leur pleine souveraineté, déterminent également une conduite de la guerre ${ }^{127}$.

Or chez Vattel, cette ambition de circonscrire la guerre par un ensemble de normes n'est pas restreinte à celle qui est externe, mais vaut également pour la guerre interne. En témoigne le remède proposé lorsqu'un soulèvement éclate : s'il se fonde sur une plainte, il faut satisfaire la requête du peuple, et, plus inattendu, s'il n'a aucun motif, il faut promettre l'amnistie aux séditieux dès qu'ils déposent les armes, et oublier ce drame. Et, laissant apparaître son attention pour la psychologie des révoltés, il affirme que cette promesse ne doit pas servir à les attaquer par surprise, car cela renforcerait leur $\operatorname{ardeur}^{128}$ et augmenterait leurs soutiens ${ }^{129}$. Contre Cicéron, Vattel défend que les promesses prises avec des insurgés doivent être tenues, faisant ainsi, comme Grotius avant lui ${ }^{130}$, du respect de son engagement -

124. Tesuya Toyoda souligne que la position de Vattel est délicate. Ce dernier est citoyen de la principauté de Neuchâtel, donc sujet du roi de Prusse, Frédéric II, auquel il offre initialement ses services. Mais cette tentative est un échec, ce qui conduit Vattel à se diriger vers le roi-électeur de Saxe. Cf. T. TESUYA, «La doctrine vattelienne de l'égalité souveraine dans le contexte Neuchâtelois », in Journal of the History of International Law, vol. 11, 2009, p. 103-124, p. 106.

125. Sur le caractère injuste de cette attaque selon Vattel voir « Lettres de Vattel à l'Avoyer et Conseil de Berne (Protestation contre l'invasion de la Saxe) Neuchâtel, 28 février 1757 », cité en annexe par E. BEGUELIN, «En souvenir de Vattel », Faculté de droit de l'Université de Neuchâtel, Recueil de travaux offert à la Société suisse des juristes à l'occasion de sa réunion à Neuchâtel 15-17 septembre 1929, Neuchâtel, 1929, note 33, p. 172. Vattel rappelle que toute offensive doit être fondée sur une plainte qui doit être portée à la connaissance du parti adverse.

126. Contre la fictive Civitas maxima de Wolff, Vattel défend une «société des nations » fondée sur la participation de chaque nation au bonheur et à la perfection des autres.

127. La coexistence des nations ne doit pas être soumise à l'arbitraire de la force.

128. Vattel considère que si le souverain ne tient pas ses engagements envers les insurgés, ceux-ci seront obligés d'entreprendre une action armée et de la mener jusqu'à son terme. Cf. E. de VAtTel, Le Droit des gens, op. cit., t. 3, III, XVIII, § 291, p. 201 : «Si ses promesses ne sont pas inviolables, il n'y aura plus de sûreté pour les rebelles à traiter avec lui ; dès qu'ils auront tiré l'épée, il faudra qu'ils en jettent le fourreau ».

129. Selon l'auteur, des spectateurs constatant le manque d'honnêteté du souverain légitime se joindront aux insurgés.

130. H. GRotius, Le Droit de la guerre et de la paix, op. cit., III, XIX, § 5-10 et II, XXIII, $\S 15$. 
acte cardinal du contractualisme en ce qu'il permet une fusion des volontés sous la forme de la parole donnée - un devoir, y compris entre un souverain et ceux qui s'opposent à lui ${ }^{131}$.

Le caractère obligatoire du respect de la parole donnée envers un corps rebelle implique de considérer ce dernier comme un contractant doté d'une volonté propre. Cela s'atteste par le concept de guerre civile proposé. Refusant la distinction entre la rébellion, "résistance ouverte et injuste », et la guerre civile, «résistance légitime » ${ }^{132}$, Vattel s'en tient à l'effet de la contestation ${ }^{133}$. L'essence de la guerre civile consiste en une scission de la nation en deux corps autonomes, qu'il s'agisse d'une république dans laquelle deux partis revendiquent chacun la représentation du tout de la société, ou d'une monarchie où s'opposent deux aspirants au trône. Il y a guerre civile dès qu'un corps matériellement en mesure de refuser la domination du souverain apparait ${ }^{134}$, provoquant de fait la division de l'État en deux entités distinctes et autonomes.

La guerre civile produit donc la scission de l'État et non sa dissolution en éléments. Avec Hobbes elle ne pouvait être qu'une guerre entre individus atomisés puisque le souverain est le seul opérateur de l'unité du peuple. Or, en s'en prenant à son souverain, un peuple redevient nécessairement une multitude désordonnée. Raison pour laquelle, selon le philosophe anglais, on ne peut dire d'un peuple qu'il s'insurge contre son souverain ${ }^{135}$. Mais si pour Vattel l'unité du peuple dérive de l'association des particuliers elle-même, alors la guerre civile est incapable d'introduire la désunion de l'état de nature. Elle est au contraire une opposition entre des groupes, et le terme

131. E. de VATtel, Le Droit des gens, op. cit., t. 3, III, XVIII, § 291, p. 201 : «Les mêmes raisons qui doivent rendre la foi des promesses inviolable et sacrée [...] de particulier à particulier, de souverain à souverain, d'ennemi à ennemi, subsistent dans toute leur force, entre le souverain et ses sujets soulevés, ou rebelles ».

132. Ibid., § 292, p. 202.

133. Sur les causes de la résistance voir Ibid. II, IV.

134. Vattel distingue également le cas d'une prise des armes des sujets contre le souverain sans que ceux-ci n'arrêtent pour autant de le reconnaître comme leur souverain. Cf. Ibid., t. 3, III, XVIII, § 295.

135. Pour Hobbes, tout discours qui affirmerait qu'un peuple s'arme contre son souverain traiterait d'une multitude confuse et non plus unie sous la forme du peuple car le peuple, identifié à l'État grâce à sa représentation par la personne du souverain, ne peut pas s'en prendre à lui-même. Ce serait une contradiction. Cf. T. HoBBES, Du Citoyen, op. cit., II, VI, p. 166 : «En effet, bien qu'on dise communément, quand éclate une insurrection de grande ampleur, que le peuple de cet État a pris les armes, ce n'est toutefois le cas que de ceux qui sont armés ou de ceux qui leur prêtent leur concours ». Et plus loin: «Toutes les fois que nous disons qu'un peuple ou une multitude veut, commande ou fait quelque chose, le mot désigne un État, qui veut, commande et agit par la volonté d'un seul homme [...]. Toutes les fois au contraire que nous disons qu'une multitude d'hommes, importante ou non, fait quelque chose sans la volonté de cet homme [...], c'est alors le fait du peuple en tant que sujet, c'est-à-dire le fait d'un ensemble de citoyens particuliers, et cela procède non pas d'une volonté singulière mais de multiples volontés de ces multiples hommes, qui sont sujets et citoyens, mais qui ne sont pas l'État ». 
«faction» n'est plus comme chez Hobbes synonyme de multiplicité mais d'opposition entre des corps constitués autour d'une volonté unique.

Pour soutenir un tel concept de guerre civile - la guerre civile comme scission de la nation en deux factions - Vattel met volontairement de côté toute considération axiologique ${ }^{136}$. Il insiste principalement sur la puissance de résistance des corps en présence, qu'il s'agisse de deux partis dont les forces contradictoires s'annulent ou d'une faction suffisamment forte pour se dresser contre l'autorité souveraine. Dans les deux cas, l'effet premier est de paralyser la capacité d'action du souverain qui, incapable de résorber cette division par une action de police, est contraint d'engager la guerre.

Si l'on s'en tient, comme Vattel, à ce qui se manifeste dès que les raisons de la guerre civile sont écartées ${ }^{137}$, alors apparaissent un ensemble d'attitudes identiques adoptées par les deux camps en présence. Des deux côtés, on trouve une tendance à se déclarer indépendant, et à refuser l'existence d'un juge supérieur commun qui soit en mesure de régler le différend. Et en plus de traiter l'autre de rebelle et de réclamer pour lui seul la représentation exclusive du corps politique, chaque camp considère son opposant comme un ennemi.

L'identité formelle des comportements des rebelles et des partisans du souverain, leur prétention identique à la liberté et l'indépendance, rend la reconnaissance du fait de leur scission nécessaire. Il s'agit d'une recommandation ponctuelle mais fondamentale; dès qu'au sein d'un État deux camps sont en mesure de se résister, il faut :

de nécessité, que ces deux partis soient considérés comme formant désormais, au moins pour un temps, deux corps séparés, deux peuples différents. Que l'un des deux ait eu tort de rompre l'unité de l'État, de résister à l'autorité légitime, ils n'en sont pas moins divisés de fait ${ }^{138}$.

Ainsi, lorsque deux entités d'un même corps se traitent en ennemis, réclament pour elles seules la souveraineté du corps politique et refusent toute subordination, alors leur séparation est effective. En conséquence, les deux camps belligérants doivent être considérés comme des égaux.

Le principe normatif de l'égalité souveraine entre les nations, que Vattel déduit de leur liberté et indépendance ${ }^{139}$, doit donc s'appliquer dans le cas

136. Ici sa stratégie est à l'inverse de traitement de la guerre dans lequel il donne une place fondamentale aux finalités de la prise des armes. En effet, les finalités de la guerre (réparation et punition) font partie de sa définition chez Vattel. Ainsi toute prise des armes n'est pas une guerre, comme la conquête et l'agression.

137. Pour une stratégie inverse voir F. BACON, Euvres philosophiques, morales et politiques, Paris, Auguste Desrez, 1838, ch. XV, «Des troubles et des séditions », p. 473-477, qui regroupe les différentes causes de sédition en causes, matérielle et efficiente, avant de leur joindre un remède adapté.

138. E. de VATTEL, Le Droit des gens, op. cit., t. 3, III, XVIII, § 293, p. 203.

139. Vattel défend la liberté et l'indépendance des nations dès les Préliminaires de son ouvrage, cf. Ibid., § 4, p. 26. 
d'une guerre civile. Et de même qu' « une petite république n'est pas moins un état souverain que le plus puissant des royaumes $\gg{ }^{140}$, de même les rebelles et les partisans du souverain sont égaux en souveraineté.

Cette recommandation n'est pas neutre, elle implique que les effets de la guerre civile soient identiques à ceux d'une guerre entre États souverains. $\mathrm{Au}$ lieu de la monstruosité de la guerre civile hobbesienne, Vattel défend la régularité de cette guerre au prix d'une identification entre guerre interne et externe : deux factions qui s'opposent sont comme « deux nations qui entrent en contestation, et qui, ne pouvant s'accorder, ont recours aux armes. » ${ }^{141}$

Ainsi, l'égalité entre les deux camps fait d'eux des hostes et non des inimicus, ce qui permet l'application du jus in bello ${ }^{142}$. De cette manière, la guerre civile se trouve circonscrite par les lois de la guerre, « ces maximes d'humanité, de modération, de droiture et d'honnêteté ${ }^{143}$. Et poursuivant l'identification entre guerre externe et interne ${ }^{144}$, Vattel affirme :

Les mêmes raisons qui en fondent l'obligation d'état à état [l'observation des lois de la guerre], les rendent autant et plus nécessaires, dans le cas malheureux où deux partis obstinés déchirent leur commune patrie ${ }^{145}$.

De la sorte Vattel fait de la guerre civile, guerre habituellement immodérée car conduite sans droit et entre des concitoyens, une guerre en forme entre ennemis, encadrée par le droit des gens. Ainsi, les factieux fondent, dès lors que le nombre leur permet de contrer la puissance du souverain, une autorité analogue à la sienne, et ce indépendamment des raisons de leur insurrection. La guerre civile permet donc, pour un temps au moins, de fonder un corps social nouveau doté d'une souveraineté propre et irréductible à celle dont le souverain en place est le gardien.

\section{Conclusion}

Les fonctions attribuées au souverain par Hobbes et Vattel, ainsi que l'étendue des droits transmis au moment du pacte, impliquent des modèles de contractualisme différents. De ces écarts découle un statut distinct accordé à la contestation. De son côté Hobbes s'efforce de construire un

140. Ibid., § 18, p. 34 .

141. Ibid.

142. Sur le jus in bello voir E. de Vattel, Le Droit des gens, op. cit., t. 3, III, VIII-X.

143. Ibid., III, XVIII, § 294, p. 203.

144. Sur l'impossibilité qu'il y a à défendre que le droit de la guerre doit s'appliquer en cas de guerre interne, tout en maintenant que la guerre en question est bien une guerre interne, voir N. Grange, De la guerre civile, Paris, A. Colin, 2009, p. 105.

145. E. de VATTEL, Le Droit des gens, op. cit., t., III, XVIII, § 294, p. 203. 
corps politique stable dont l'autorité est incontestable. En conséquence son contractualisme repose sur l'abandon d'un droit absolu, grâce une décision du particulier qui se veut un acte ultime de sa volonté : il ne peut se dédire. Dans cette perspective, la contestation du souverain est nécessairement la récupération illégale d'un droit par ailleurs incompatible avec le maintien de la paix. À l'inverse, chez Vattel, le souverain n'a qu'une fonction contingente. Il ne sert qu'à faciliter la réalisation du projet originaire de toute association humaine. Le corps politique est constitué et dispose de la souveraineté par la simple association des particuliers. Dans ces conditions, la dissension exprime une insatisfaction face aux bienfaits des actions du souverain. Et bien que Vattel refuse qu'un sujet s'attribue lui-même le droit de priver la nation de son souverain ${ }^{146}$, son contractualisme le conduit à restreindre la puissance du souverain à l'autorité qui l'établit. Ainsi, la nation peut, lorsque le souverain s'est mis en état de guerre avec elle, s'extraire du lien d'obligation qui la lie à lui et le traiter en ennemi.

Il s'ensuit que pour Hobbes, la dissension, lorsqu'elle prend la forme d'une sédition dont l'action conduit à la guerre civile, fait disparaître la concorde et met en péril la société politique toute entière. Le rebelle est responsable du pire des crimes puisqu'il fait apparaître l'état de nature au sein de l'état civil, raison pour laquelle le souverain doit lui faire la guerre. Ainsi, les insurgés doivent être traités comme des ennemis. Contre le danger de la sédition Hobbes préconise donc l'absoluité de la souveraineté, qui s'accompagne chez lui d'un refus théorique de la distinction entre les régimes de formes bonnes et dégénérées. La tyrannie n'est que le nom que les mécontents donnent à la monarchie ${ }^{147}$. Ainsi, on ne peut nommer le souverain tyran pour justifier son renversement.

Or pour Vattel, lorsque la dissension prend la forme d'une contestation dont la puissance de résister affiche les mêmes traits que la souveraineté confiée au souverain - liberté et indépendance - il est nécessaire d'admettre l'apparition d'un nouveau corps politique. Raison pour laquelle il faut mettre de côté les raisons de l'insurrection pour ne tenir compte que des aptitudes à l'autonomie, car en elles se trouvent les germes de souverainetés nouvelles. Et bien que Vattel préconise comme Hobbes qu'il faille faire la guerre aux rebelles, la conséquence qu'il tire est inverse. Puisqu'il défend l'égalité souveraine des corps politiques à partir de leur capacité à se gouverner par eux-mêmes, Vattel fait de la guerre que le souverain mène contre les rebelles une relation qui atteste leur souveraineté.

146. E. de VATTEL, Le Droit des gens, op. cit., t. 1, I, IV, § 50.

147. T. HobBes, Léviathan, op. cit., II, XIX, p. 306. 